\title{
Differential Radiometers Using Fabry-Perot Interferometric Technique for Remote Sensing Determination of Various Atmospheric Trace Gases
}

\author{
Georgieva, E.M ${ }^{1}$, Heaps, W.S. ${ }^{2}$, Wilson, E.L. ${ }^{2}$ \\ ${ }^{1}$ Goddard Earth Sciences and Technology Center, UMBC, Baltimore, MD 21228, USA \\ e-mail: egeorgie@pop500.gsfc.nasa.gov \\ ${ }^{2}$ NASA, Goddard Space flight Center, Greenbelt, MD 20771, USA
}

New type of remote sensing instrument based upon the Fabry-Perot interferometric technique has been developed at NASA's Goddard Space Flight Center. Fabry-Perot interferometry (FPI) is a well known, powerful spectroscopic technique and one of its many applications is to be used to measure greenhouse gases and also some harmful species in the atmosphere. With this technique, absorption of particular species is measured and related to its concentration. A solid Fabry-Perot etalon is used as a frequency filter to restrict the measurement to particular absorption bands of the gas of interest. With adjusting the thickness of the etalon that separation (in frequency) of the transmitted fringes can be made equal to the almost constant separation of the gas absorption lines. By adjusting the temperature of the etalon, which changes the index of refraction of its material, the transmission fringes can be brought into nearly exact correspondence with absorption lines of the particular species. With this alignment between absorption lines and fringes, changes in the amount of a species in the atmosphere strongly affect the amount of light transmitted by the etalon and can be related to gas concentration.

The instrument that we have developed detects the absorption of various atmospheric trace gases in direct or reflected sunlight. Our instrument employing FabryPerot interferometer makes use of two features to achieve high sensitivity. The first is high spectral resolution enabling one to match the width of an atmospheric absorption feature by the instrumental band pass. The second is high optical throughput enabled by using multiple spectral lines simultaneously. For any species that one wishes to measure, this first feature is available while the use of multiple spectral features can be employed only for species with suitable spectra and freedom from interfering species in the same wavelength region. We have developed an instrument for use as ground based, airborne and satellite sensor for gases such as carbon dioxide $(1570 \mathrm{~nm})$, oxygen $(762 \mathrm{~nm}$ and 768 $\mathrm{nm}$ lines sensitive to changes in oxygen pressure and oxygen temperature) and water vapor $(940 \mathrm{~nm})$. Our current goal is to develop an ultra precise, inexpensive, ground based device suitable for wide deployment as a validation instrument for the Orbiting Carbon Observatory (OCO) satellite. We show sensitivity measurements for $\mathrm{CO}_{2}, \mathrm{O}_{2}$, and $\mathrm{H}_{2} \mathrm{O}$, compare our measurements to those obtained using other types of sensors and discuss some of the peculiarities that must be addressed in order to provide the very high quality column detection required for solving problems about global distribution of greenhouse gases and climatological models.

The instrument setup is shown in Fig. 1.The incident light in the $\mathrm{CO}_{2}$ channel is pre-filtered at central wavelength of $1570 \mathrm{~nm}$. The light then splits between the FabryPerot and Reference sub-channels with $90 \%$ of the light going to the Fabry-Perot sub- 
channel. In the Fabry-Perot sub-channel the light passes through the Fabry-Perot etalon mounted in a temperature controlled oven for fine FSR (free spectral range) tuning. Set of off axis parabolic (OAP) gold mirrors focuses light onto InGaAs detectors for the $\mathrm{CO}_{2}$ sub-channels and Si detectors for the two oxygen channels. For bringing light into the instrument we use fiber bundles, which intentionally scrambled the incident light to remove spatial information.

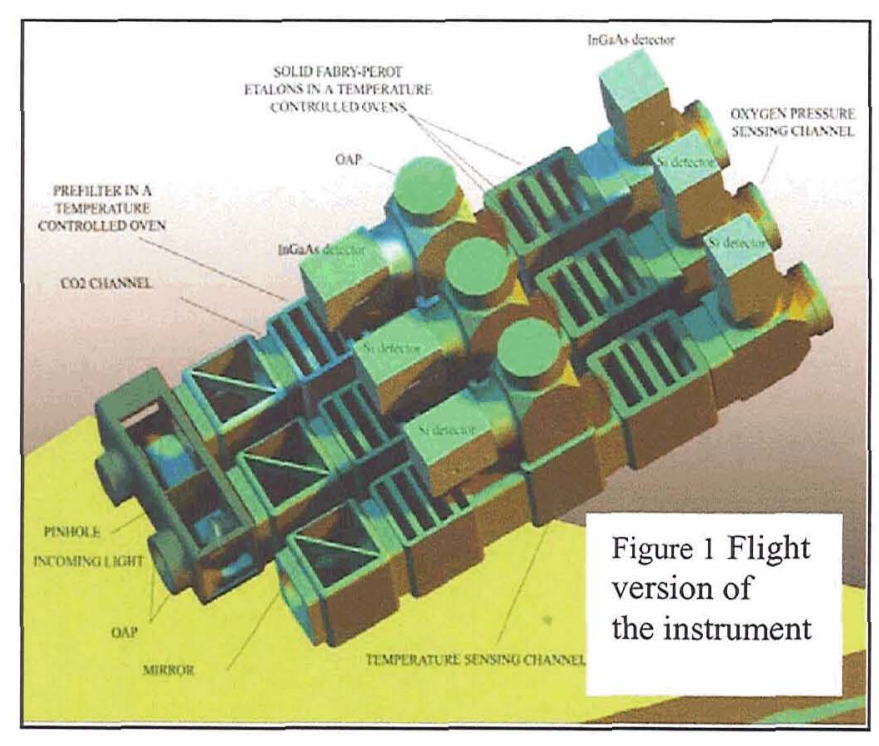

The flight hardened version of the instrument was tested at two separate flight campaigns: at NASA Dryden Research Center and at New Hampshire Polar Aura Validation campaign on NASA's DC-8 research airplane.

In addition to laboratory validation of the FPI instrument, we continuously perform daily ground based measurements of the actual atmospheric column in a variety of atmospheric conditions using a fiber coupled sun tracker. The initial tests indicate that the instrument can detects changes in the $\mathrm{CO}_{2}$ column as small as $2.3 \mathrm{ppm}$ with a one second average and better than $1 \mathrm{ppm}$ in less than 10 seconds averaging capability. The airborne instrument using light reflected off the ground has a precision of about $2 \%$. The detection limit for the oxygen column pressure changes is as small as $0.88 \mathrm{mbar}$. Water vapor has been measured in the lab and from the ground. The precision is a few percent for one second average.

The instrument is characterized by high signal to noise ratio, fast response and great specificity. We have performed simulations and instrument designs for systems to detect also $\mathrm{CO},{ }^{13} \mathrm{CO}_{2}, \mathrm{CH}_{4}, \mathrm{CH}_{2} \mathrm{O}, \mathrm{NH}_{3}, \mathrm{SO}_{2}, \mathrm{~N}_{2} \mathrm{O}, \mathrm{NO}_{2}$, and $\mathrm{O}_{3}$. Our most recent laboratory data for a new ${ }^{13} \mathrm{CO}_{2}$ channel at $2.1 \mu \mathrm{m}$ intended to measure the carbon isotope ratio in the Mars' atmosphere are also presented. Isotope ratios of carbon and oxygen are important for understanding the evolution of the Martian atmosphere and the history of volatiles on Mars. The current existing measurements for the atmospheric $\mathrm{CO}_{2}$ isotope ratios show a large scatter and uncertainties.

In another area of research we are interested in developing a small-size channel for $\mathrm{CO}_{2}$ capable of doing simultaneous measurements with the AERONET (Aerosol Robotic Network) at NASA, Goddard to study the hypothesis that atmospheric aerosols affect the regional terrestrial carbon cycle.

We present recent data from our ground based measurements of $\mathrm{O}_{2}, \mathrm{CO}_{2}, \mathrm{H}_{2} \mathrm{O}$ and ${ }^{13} \mathrm{CO}_{2}$ and discuss extension of the technique to new species and applications. 